\title{
Sensitivity Invariants for Linear Time-Invariant Networks
}

\author{
M. N. S. SWAMY, CHAMPA BHUSHAN, AND K. THULASIRAMAN
}

\begin{abstract}
For a general linear time-invariant network it is shown that the sum of the sensitivities, of any order, of a network function over different parameter sets is invariant. An expression is derived for calculating the sum of $(k+1)$ th-order sensitivities knowing the same for $k$ th-order sensitivities. It is also shown that lower bounds for the quadratic sensitivity index may be obtained by using the firstorder sensitivity invariants.
\end{abstract}

\section{INTRODUCTION}

$\mathrm{T}$ 7 HE CONJECTURE of Leeds and Ugron [1] that the sum of the sensitivity functions (over all components of each continuously equivalent network) is invariant, with respect to various equivalent networks, has led to a number of investigations on the invariant nature of the sum of the sensitivities of a network function. A good survey of these investigations is available in [2], [3]. Recently, Sablatash and Seviora [4] have used Tellegen's theorem and the concept of the adjoint or transpose of a network [5]-[7] to establish the invariant nature of the sum of the sensitivities of network functions of a certain class of networks containing uniformly distributed $\overline{R C}$ and lossless lines. Similar invariance results may be established for networks that in addition include active elements, as well as tapered lines, by either following Blostein's approach [8] or the adjoint approach [9]. We first briefly summarize these results, show how these may. be used to obtain lower bounds for Schoefler's [10] quadratic sensitivity index, and then establish the invariant nature of the sum of the higher order sensitivities of network functions over different sets of parameters.

\section{Sensitivity Invariants and Quadratic SENSITIVITY INDEX}

Consider a general $n$-port network $N$ consisting of lumped resistors $\left(R_{i}=1 / G_{i}\right)$, inductors $\left(L_{i}=1 / \Gamma_{i}\right)$, capacitors $\left(C_{i}=1 / D_{i}\right)$, gyrators, controlled sources [current to voltage transducers (CVT's), voltage to current transducers (VCT's), voltage to voltage transducers (VVT's), and current to current transducers (CCT's) ], negative impedance converters (NIC's), lossless tapered lines, and $\overline{R C}$ tapered lines. Let the impedance matrix of a gyrator be denoted by

Manuscript received July 9, 1971; revised April 14, 1972. This work was supported by the National Research Council of Canada under Grant A-7739. This paper is part of a dissertation submitted by C. Bhushan to Sir George Williams University, Montreal, Que., Canada, in partial fulfillment of the requirements for the D.Eng. degree.

M. N. S. Swamy and C. Bhushan are with the Department of Electrical Engineering, Sir George Williams University, Montreal, Que., Canada.

$\mathrm{K}$. Thulasiraman is with the Department of Electrical Engineering, Indian Institute of Technology, Madras, India.

$$
\left[\begin{array}{ll}
0 & \alpha_{i 1} \\
\alpha_{i 2} & 0
\end{array}\right]
$$

and the parameters of a nonuniform transmission line (NUTL) be given as follows:

$$
\begin{aligned}
& \bar{Z}_{i}(s, x)=Z_{0 i}(s) f(x) \\
& \bar{Y}_{i}(s, x)=Y_{0 i}(s) g(x), \quad a \leq x \leq b
\end{aligned}
$$

where $\bar{Z}_{i}(s, x)$ and $\bar{Y}_{i}(s, x)$ are series impedance per unit length and shunt admittance per unit length of a general NUTL. A lossless NUTL is described by

$$
\begin{aligned}
& \bar{L}_{i}=L_{0 i} f(x) \\
& \bar{C}_{i}=C_{0 i} g(x), \quad a \leq x \leq b
\end{aligned}
$$

and a tapered $\overline{R C}$ line by

$$
\begin{aligned}
& \bar{R}_{i}=R_{0 i} f(x) \\
& \bar{C}_{i}=C_{0 i} g(x), \quad a \leq x \leq b .
\end{aligned}
$$

Let

$$
D_{0 i}=1 / C_{0 i}
$$

and

$$
Z_{\overline{L C} i}=\sqrt{\frac{L_{0 i}}{C_{0 i}}}=\frac{1}{Y_{\overline{L C} i}} \text { and } Z_{\overline{R C} i}=\sqrt{\frac{R_{0 i}}{C_{0 i}}}=\frac{1}{Y_{\overline{R C} i}}
$$

be the "characteristic impedance" of a lossless NUTL and "characteristic impedance" of a tapered $\overline{R C}$ line, respectively. Further, let $r_{i}=1 / g_{i}$ and $\delta_{i}=1 / \gamma_{i}$ be the transfer resistance of a CVT and transfer conductance of a VCT, respectively.

For the network $N$, if $p_{k}$ is a member of the set,

$P=\left\{p_{k}\right\}=\left\{R_{i}, L_{i}, D_{i},\left(\alpha_{i 1}, \alpha_{i 2}\right),\left(R_{0 i}, D_{0 i}\right), Z_{\overline{L C} i}, r_{i}, \gamma_{i}\right\}$

where the subset $\left(R_{0 i}, D_{0 i}\right)$ may be replaced by $Z_{\overline{R C_{i}}}$, while the subset $Z_{\overline{L C}}$ by $\left(L_{0 i}, D_{0 i}\right)$; then we have [9]

$$
\begin{aligned}
\sum_{p_{k}} \frac{p_{k}}{Z_{i j}} \frac{\partial Z_{i j}}{\partial p_{k}} & =1 \\
\sum_{p_{k}} \frac{p_{k}}{Y_{i j}} \frac{\partial Y_{i j}}{\partial p_{k}} & =-1 \\
\sum_{p_{k}} p_{k} \frac{\partial T}{\partial p_{k}} & =0 \\
\sum_{p_{k}} p_{k} \frac{\partial S}{\partial p_{k}} & =\frac{1}{2}\left(U-S^{2}\right) \\
\sum_{p_{k^{\prime}}} p_{k^{\prime}} \frac{\partial S}{\partial p_{k}} & =0
\end{aligned}
$$


where $Z_{i j}$ is the transfer impedance between the $i$ th and $j$ th ports of $N, Y_{i j}$ is the transfer admittance, $T$ is the open-circuit voltage ratio or short-circuit current ratio, $S$ is the scattering matrix, $U$ is the unit matrix, and $\left\{p_{k}{ }^{\prime}\right\}=\left\{p_{k}\right\} \cup\left\{\rho_{i}\right.$, the normalizing resistors $\}$.

It can also be shown [9] that for the network $N$, over the subset $M=\left\{m_{k}\right\}=\left\{L_{i}, C_{i},\left(L_{0 i}, C_{0 i}\right)\right\}$, the summed sensitivity of a network function $F$ is given by

$$
\sum_{m_{k}} \frac{m_{k}}{F} \frac{\partial F}{\partial m_{k}}=\frac{s}{F} \frac{\partial F}{\partial s} .
$$

For a network $N_{1}$ consisting of lumped resistors, capacitors, gyrators, $\overline{R C}$ tapered lines, controlled sources, and NIC's, if $q_{k}$ is a member of the set $Q=\left\{q_{k}\right\}$ $=\left\{R_{i}, C_{i},\left(\alpha_{i 1}, \alpha_{i 2}\right),\left(R_{0 i}, C_{0 i}\right), r_{i}, \gamma_{i}\right\}$, then it can be shown that [9]

$$
\begin{aligned}
\sum_{q_{k}} \frac{q_{k}}{Z_{i j}} \frac{\partial Z_{i j}}{\partial q_{k}} & =\frac{2 s}{Z_{i j}} \frac{\partial Z_{i j}}{\partial s}+1 \\
\sum_{q_{k}} \frac{q_{k}}{Y_{i j}} \frac{\partial Y_{i j}}{\partial q_{k}} & =\frac{2 s}{Y_{i j}} \frac{\partial Y_{i j}}{\partial s}-1 \\
\sum_{q_{k}} \frac{q_{k}}{T} \frac{\partial T}{\partial q_{k}} & =\frac{2 s}{T} \frac{\partial T}{\partial s} \\
\sum_{q_{k}} q_{k} \frac{\partial S}{\partial q_{k}} & =2 s \frac{\partial S}{\partial s}+\frac{1}{2}\left(U-S^{2}\right) \\
\sum_{\boldsymbol{q}_{k^{\prime}}} q_{k^{\prime}} \frac{\partial S}{\partial q_{k^{\prime}}} & =2 s \frac{\partial S}{\partial s}
\end{aligned}
$$

where $\left\{q_{k}{ }^{\prime}\right\}=\left\{q_{k}\right\} \cup\left\{\rho_{i}\right\}$.

Using the approach of Kasper and Schmidt [11], the different invariances given in this section may be used as constraints in minimizing the quadratic sensitivity index

$$
\phi=\sum_{p_{k}}\left|S_{p_{k}}^{F}\right|^{2}
$$

where

$$
S_{p_{k}}^{F}=\frac{p_{k}}{F} \frac{\partial F}{\partial_{p_{k}}} .
$$

For the different networks considered in this section, some of the lower bounds are as follows.

1) For network $N$,

$$
\phi=\sum_{p_{k}}\left|S_{p_{k}}^{F}\right|^{2} \geq \frac{1}{n}
$$

where $F$ is $Z_{i j}$ or $Y_{i j}$ and $n$ is the total number of elements in the set $\left\{p_{k}\right\}$.

2) For network $N_{1}$,

$$
\phi=\sum_{a_{k}}\left|S_{q_{k}}{ }^{Z_{i j}}\right|^{2} \geq \frac{1}{n_{1}}\left|S_{8} Z_{i j}+1\right|^{2}+\frac{1}{n_{2}}\left|S_{s}{ }^{Z_{i j}}\right|^{2}
$$

$$
\begin{aligned}
& \phi=\sum_{q_{k}}\left|S_{q_{k}} Y_{i j}\right|^{2} \geq \frac{1}{n_{1}}\left|S_{s} Y_{i j}-1\right|^{2}+\frac{1}{n_{2}}\left|S_{s} Y_{i j}\right|^{2} \\
& \phi=\sum_{q_{k}}\left|S_{q_{k}}{ }^{T}\right|^{2} \geq\left(\frac{1}{n_{1}}+\frac{1}{n_{2}}\right)\left|S_{s}{ }^{T}\right|^{2}
\end{aligned}
$$

where $n_{1}$ and $n_{2}$ are the number of elements in the sets $\left\{q_{k 1}\right\}=\left\{R_{i},\left(\alpha_{i 1}, \alpha_{i 2}\right), R_{0 i}, r_{i}, \gamma_{i}\right\}$ and $\left\{q_{k 2}\right\}=\left\{C_{i}, C_{0 i}\right\}$, respectively.

3) For a network $\mathrm{N}_{2}$ consisting of lumped capacitors, inductors, lossless tapered lines, VVT's, CCT's and NIC's,

$$
\begin{aligned}
& \phi=\sum_{m_{k}}\left|S_{m_{k}}{ }^{Z_{i j}}\right|^{2} \geq \frac{1}{4 n_{1}}\left|S_{s}{ }^{Z_{i j}}-1\right|^{2}+\frac{1}{4 n_{2}}\left|S_{8}{ }^{Z_{i j}}+1\right|^{2} \\
& \phi=\sum_{m_{k}}\left|S_{m_{k}}{ }^{Y_{i j}}\right|^{2} \geq \frac{1}{4 n_{1}}\left|S_{8} Y_{i j}+1\right|^{2}+\frac{1}{4 n_{2}}\left|S_{s} Y_{i j}-1\right|^{2} \\
& \phi=\sum_{m_{k}}\left|S_{m_{k}}{ }^{T}\right|^{2} \geq \frac{1}{4}\left(\frac{1}{n_{1}}+\frac{1}{n_{2}}\right)\left|S_{s}{ }^{T}\right|^{2}
\end{aligned}
$$

where $n_{1}$ and $n_{2}$ are, respectively, the number of elements in $\left\{m_{k 1}\right\}=\left\{C_{i}, C_{0 i}\right\}$ and $\left\{m_{k 2}\right\}=\left\{L_{i}, L_{0 i}\right\}$.

It may be observed from the above expressions that as the number of elements is increased, the lower bounds decrease, thereby strengthening the conjecture of Lccds and Ugron [1].

\section{Higher Order Sensitivity Invariants}

We now establish the invariant nature of the sum of the second-order sensitivities of network functions.

For the network $N$, over the set $\left\{p_{k}\right\}$ from (1),

$$
\boldsymbol{Z}=\sum_{k=1}^{n} p_{k} \frac{\partial \boldsymbol{Z}}{\partial p_{k}} .
$$

In writing the above expression it is assumed that the set $\left\{p_{k}\right\}$ consists of $n$ elements $p_{k}, k=1,2, \cdots, n$.

From (12),

$$
\begin{aligned}
& \sum_{i=1}^{n} p_{i} \frac{\partial Z}{\partial p_{i}}=\sum_{i=1}^{n} \sum_{k=1}^{n} p_{i} p_{k} \frac{\partial^{2} Z}{\partial p_{i} \partial p_{k}} \\
&+\sum_{i=1}^{n} \sum_{k=1}^{n} p_{i} \frac{\partial}{\partial p_{i}}\left(p_{k}\right) \frac{\partial Z}{\partial p_{k}} .
\end{aligned}
$$

Since

$$
\frac{\partial p_{k}}{\partial p_{i}}= \begin{cases}0, & \text { if } i \neq k \\ 1, & \text { if } i=k\end{cases}
$$

it is readily seen that the second term on the right-hand side of (13) is equal to $\sum_{i=1}^{n} p_{i}\left(\partial \boldsymbol{Z} / \partial p_{i}\right)$. Thus it follows from (13) that

$$
\sum_{i=1}^{n} \sum_{k=1}^{n} p_{i} p_{k} \frac{\partial^{2} Z}{\partial p_{i} \partial p_{k}}=0
$$

That is, the sum of second-order sensitivities of the impedance matrix $Z$ of the network $N$ is invariant over the set $\left\{p_{k}\right\}$. 
For the admittance matrix $Y$, current or voltage transfer function $T$, and scattering matrix $S$, it can be shown that

$$
\begin{aligned}
& \sum_{i=1}^{n} \sum_{k=1}^{n} p_{i} p_{k} \frac{\partial^{2} Y}{\partial p_{i} \partial p_{k}}=2 Y \\
& \sum_{i=1}^{n} \sum_{k=1}^{n} p_{i} p_{k} \frac{\partial^{2} T}{\partial p_{i} \partial p_{k}}=0 \\
& \sum_{i=1}^{n} \sum_{k=1}^{n} p_{i} p_{k} \frac{\partial^{2} S}{\partial p_{i} \partial p_{k}}=-\frac{1}{2}(U+S)\left(U-S^{2}\right)(17) \\
& \sum_{p_{i}{ }^{\prime} \in\left\{p_{k^{\prime}}\right\}} \sum_{p_{k^{\prime}} \in\left\{p_{k^{\prime}}\right\}} p_{i^{\prime}}{ }^{\prime} p_{k}{ }^{\prime} \frac{\partial^{2} S}{\partial p_{i}^{\prime} \partial p_{k}^{\prime}}=0
\end{aligned}
$$

Similarly, it can be shown that for the network $N$, over the set $\left\{m_{k}\right\}$,

$$
\sum_{i=1}^{n} \sum_{k=1}^{n} m_{i} m_{k} \frac{\partial^{2} F}{\partial m_{i} \partial m_{k}}=s^{2} \frac{\partial^{2} F}{\partial s^{2}}
$$

where $m_{i}$ and $m_{k}$ are the members of the set $\left\{m_{k}\right\}$ and $F$ is an impedance or admittance parameter or voltage or current transfer function. The same relationship holds good even if $F$ is a $\boldsymbol{Z}$ - or $\boldsymbol{Y}$-matrix.

Consider next the network $N_{1}$, over the set $\left\{q_{k}\right\}$. Let the set $\left\{q_{k}\right\}$ consist of $n$ elements $q_{k}, k=1,2, \cdots, n$. From (7),

$$
\sum_{k=1}^{n} q_{k} \frac{\partial \boldsymbol{Z}}{\partial q_{k}}=2 s \frac{\partial \boldsymbol{Z}}{\partial s}+\boldsymbol{Z} .
$$

Following the same procedure as outlined earlier, the following can be proved using (7)-(11):

$$
\begin{gathered}
\sum_{i=1}^{n} \sum_{k=1}^{n} q_{i} q_{k} \frac{\partial^{2} Z}{\partial q_{i} \partial q_{k}}=6 s \frac{\partial Z}{\partial s}+4 s^{2} \frac{\partial^{2} Z}{\partial s^{2}} \\
\sum_{i=1}^{n} \sum_{k=1}^{n} q_{i} q_{k} \frac{\partial^{2} Y}{\partial q_{i} \partial q_{k}}=4 s^{2} \frac{\partial^{2} Y}{\partial s^{2}}-2 s \frac{\partial Y}{\partial s}+2 Y \\
\sum_{i=1}^{n} \sum_{k=1}^{n} q_{i} q_{k} \frac{\partial^{2} T}{\partial q_{i} \partial q_{k}}=2 s \frac{\partial T}{\partial s}+4 s^{2} \frac{\partial^{2} T}{\partial s^{2}} \\
\sum_{i=1}^{n} \sum_{k=1}^{n} q_{i} q_{k} \frac{\partial^{2} S}{\partial q_{i} \partial q_{k}}=4 s^{2} \frac{\partial^{2} S}{\partial s^{2}}+2 s \frac{\partial S}{\partial s}-4 s \\
\cdot S \frac{\partial S}{\partial s}-\frac{1}{2}\left(U-S^{2}\right)-\frac{S}{2}\left(U-S^{2}\right) \\
\sum_{q_{i} i^{\prime} \in\left\{q_{k^{\prime}}\right\}} \sum_{q_{k^{\prime}} \in\left\{q_{k^{\prime}}\right\}} q_{i}{ }^{\prime} q_{k}{ }^{\prime} \frac{\partial^{2} S}{\partial q_{i}{ }^{\prime} \partial q_{k}{ }^{\prime}}=4 s^{2} \frac{\partial^{2} S}{\partial s^{2}}+2 s \frac{\partial S}{\partial s} .
\end{gathered}
$$

We conclude this section with a general result that will help in obtaining the sum of the $(k+1)$ th-order sensitivities of a network function from the sum of the $k$ th-order sensitivities.

Consider any network function $F\left(p_{1}, p_{2}, \cdots, p_{n}\right)$. Let $X_{k}$ be the $k$ th-order summed sensitivity and $X_{k+1}$ the $(k+1)$ th-order summed sensitivity; then

$$
\begin{array}{r}
X_{k}=\sum_{i_{k}=1}^{n} \sum_{i_{k=1}=1}^{n} \cdots \sum_{i_{2}=1}^{n} \sum_{i_{1}=1}^{n} p_{i 1} p_{i 2} \cdots p_{i k} \\
\frac{\partial^{k} F}{\partial p_{i 1} \partial p_{i 2} \cdots \partial p_{i k}}
\end{array}
$$

and

$$
\begin{array}{r}
X_{k+1}=\sum_{i_{k+1}=1}^{n} \sum_{i_{k}=1}^{n} \cdots \sum_{i_{2}=1}^{n} \sum_{i_{1}=1}^{n} p_{i 1} p_{i 2} \cdots p_{i k+1} \\
\frac{\partial^{k+1} F}{\partial p_{i 1} \partial p_{i 2} \cdots \partial p_{i k+1}}
\end{array}
$$

From (26),

$$
\begin{gathered}
\sum_{i_{k+1}=1}^{n} p_{i k+1} \frac{\partial X_{k}}{\partial p_{i k+1}}=X_{k+1}+\sum_{i_{k}=1}^{n} \sum_{i_{k-1}=1}^{n} \cdots \sum_{i_{2}=1}^{n} \sum_{i_{1}=1}^{n} \\
\frac{\partial^{k} F}{\partial p_{i 1} \partial p_{i 2} \cdots \partial p_{i k}} \sum_{i_{k+1}=1}^{n} p_{i k+1} \frac{\partial\left(p_{i 1} p_{i 2} \cdots p_{i k}\right)}{\partial p_{i k+1}} .
\end{gathered}
$$

In the second term of the right-hand side of (28), $k$ parameters are taken at a time, but some of the parameters may occur more than once. Let a typical term of $\left(p_{i 1} p_{i 2} \cdots p_{i k}\right)$ be

$$
p_{i 1}^{x_{i 1}} p_{i 2}^{x_{i 2}} \cdots p_{i k}^{x_{i k}} \triangleq A, \quad i j \in\{1,2, \cdots, n\} .
$$

It should be noted that $\sum_{j=1}^{k} x_{i j}=k$. Then,

$$
p_{i j} \frac{\partial}{\partial p_{i j}}(A)= \begin{cases}0, & \text { if } x_{i j}=0 \\ x_{i j} A, & \text { if } x_{i j} \neq 0 .\end{cases}
$$

Hence

$$
\sum_{j=1}^{n} p_{i j} \frac{\partial(A)}{\partial p_{i j}}=A \sum_{j=1}^{k} x_{i j}=k A .
$$

Using (31) in (28),

$$
\sum_{i_{k+1}=1}^{n} p_{i k+1} \frac{\partial X_{k}}{\partial p_{i k+1}}=X_{k+1}+k X_{k}
$$

or

$$
X_{k+1}=\sum_{i=1}^{n} p_{i} \frac{\partial X_{k}}{\partial p_{i}}-k X_{k} .
$$

Equation (32) expresses the $(k+1)$ th-order summed sensitivity in terms of the summed $k$ th-order sensitivity.

\section{REFERENCES}

[1] J. V. Leeds, Jr., and G. I. Ugron, "Simplified multiple parameter sensitivity calculations and continuously equivalent networks," IEEE Trans. Circuit Theory, vol. CT-14, pp. 188-191, June 1967.

[2] W. J. Butler and S. S. Haykin, "Multiparameter sensitivity problems in network theory," Proc. Inst. Elec. Eng., vol. 117, no. 12 , pp. 2228-2235, Dec. 1970.

[3] S. R. Parker, "Sensitivity: Old questions, some new answers," IEEE Trans. Circuit Theory (Special Issue on Computer-Aided Circuit Design), vol. CT-18, pp. 27-35, Jan. 1971.

[4] M. Sablatash and R. Seviora, "Sensitivity invariants for scatter- 
ing matrices," IEEE Trans. Circuit Theory (Corresp.), vol. CT18, pp. 282-284, Mar. 1971.

[5] S. W. Director and R. A. Rohrer, "Automated network designThe frequency-domain case," IEEE Trans. Circuit Theory, vol. CT-16, pp. 330-337, Aug. 1969.

[6] - . "The generalized adjoint network and network sensitivities," IEEE Trans. Circuit Theory, vol. CT-16, pp. 318-323, Aug. 1969.

[7] B. B. Bhattacharyya and M. N.S. Swamy, "Network transposition and its application in synthesis," IEEE Trans. Circuit Theory (Corresp.), vol. CT-18, pp. 394-397, May 1971.

[8] M. L. Blostein, "Sensitivity analysis of parasitic effects on resistance-terminated LC two-ports," IEEE Trans. Circuit Theory, vol. CT-14, pp. 21-25, Jan. 1967.

[9] M. N. S. Swamy, C. Bhushan, and K. Thulasiraman, "Sensitivity invariants for active lumped-distributed networks," Electron. Lett., vol. 8, no. 2, pp. 26-27, 1972.

[10] J. D. Schoeffler, "The synthesis of minimum sensitivity networks," IEEE Trans. Circuit Theory, vol. CT-11, pp. 271-276, June 1964.

[11] G.Schmidt and R. Kasper, "On minimum sensitivity networks," IEEE Trans. Circuit Theory (Corresp.), vol. CT-14, pp. 438440, Dec. 1967.

[12] K. Geher and J. Solymosi, "Calculation of higher-order sensitivities and higher-order sensitivity invariants," in Proc. IEEE Int. Symp. Network Theory, pp. 7-8, 1971.

\title{
Single-Transistor All-Pass Networks
}

\author{
HARVEY RUBIN AND REED K. EVEN
}

\begin{abstract}
A network configuration consisting of a resistive twoport bridged by a lossless one-port is considered. It is shown that in certain cases this configuration is capable of realizing a voltage transfer function equal to that of a constant-resistance passive lattice. As a special case, conditions are derived on the two-port parameters for the structure to be all pass. It is determined that a single transistor suffices to realize the two-port network, thus resulting in a very simple all-pass structure; only one coil, one capacitor, and one transistor are necessary to realize a second-degree all-pass voltage transfer function. Higher order transfer functions can be realized by adding an $L C$ branch per each additional second-order factor to the same transistor circuit. In the second-order case, losses in the coil can be compensated. In higher order realizations coil losses cannot be compensated, but a design technique which achieves an arbitrarily close approximation to the desired all-pass transfer.function is presented. Methods of cascading lower order sections are discussed and experimental results are given.
\end{abstract}

\section{INTRODUCTION}

M ODERN more stringent requirements of communication networks, such as increasing their bandwidth, pose special problems in the design of phase equalizers.

Passive realizations suffer from many drawbacks, the most important of which are 1) an excessive number of coils and capacitors, 2) lack of unbalanced transformerless sections for certain values of pole $Q[1]$, and 3 ) finite component $Q$ 's make it impossible to conform to stringent specifications on the amplitude characteristic.

Realizations using active elements, namely, transistors in operational amplifiers [2] or gyrators [3], wherein the active components determine the locations of the poles and zeros, can be used over a limited fre-

Manuscript received November 5, 1971; revised June 12, 1972. The authors are with Bell Laboratories, Holmdel, N. J. quency range only. The bandwidth limitation is due in this case to the large number of transistors needed in the implementation of the operational amplifier or the gyrator.

A circuit which overcomes to a certain extent the bandwidth limitation is being developed at this time [4], but it needs one differential input amplifier of a high common-mode rejection ratio.

Two realization methods requiring only one transistor may be found in the literature. The circuit proposed by Allemandou [5] suffers from limited bandwidth and accuracy due to the fact that its performance depends on the emitter and collector voltages being equal in magnitude and opposite in phase.

Aronhime [6] describes only one application of the single-transistor circuit, namely, its use as a first-order all-pass network. It is shown in this paper that this circuit can be used for the realization of any transfer function that can be realized by a passive lattice. The method presented leads to considerable savings in the number of reactive components; it enables the designer to compensate for the inevitable losses in these components and results in a broad-band realization of the desired transfer function.

\section{The General Configuration}

Consider a configuration consisting of a resistive unbalanced two-port $N_{a}$ with the short-circuit admittance matrix

$$
Y_{a}=\left[\begin{array}{ll}
G_{1} & G_{2} \\
G_{3} & G_{4}
\end{array}\right]
$$

bridged by a one-port $N_{b}$ of admittance $Y$. The voltage 